\title{
Development
}

\section{Heparan Sulfated Glypican-4 Is Released from Astrocytes by Proteolytic Shedding and GPI- Anchor Cleavage Mechanisms}

\author{
Kevin Huang, ${ }^{1,2}$ and ${ }^{D S}$ Sungjin Park ${ }^{1}$ \\ https://doi.org/10.1523/ENEURO.0069-21.2021
}

${ }^{1}$ Department of Neurobiology, University of Utah School of Medicine, Salt Lake City, UT 84112 and ${ }^{2}$ Interdepartmental Program in Neuroscience, University of Utah, Salt Lake City, UT 84112

\section{Visual Abstract}

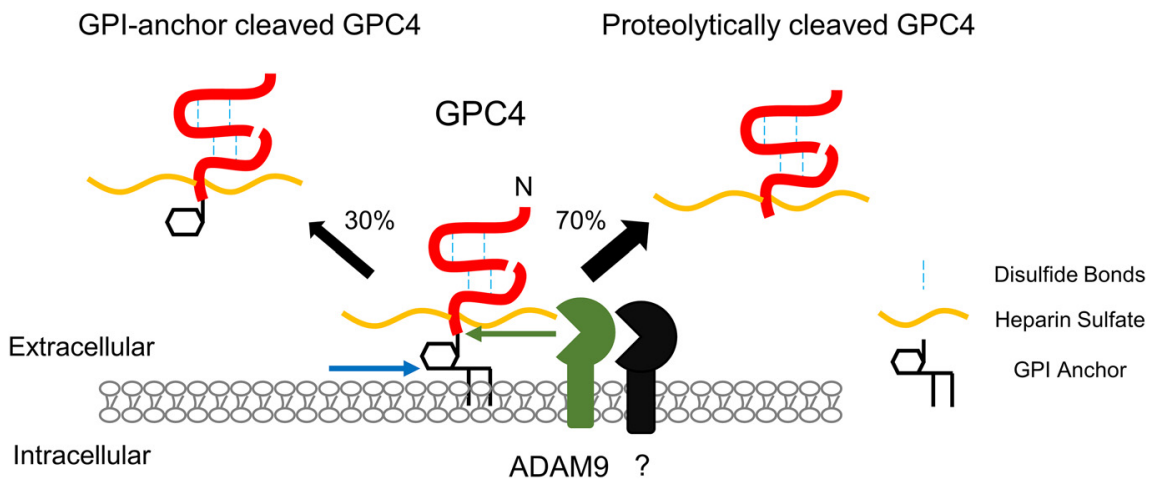

Astrocytes provide neurons with diffusible factors that promote synapse formation and maturation. In particular, glypican-4/GPC4 released from astrocytes promotes the maturation of excitatory synapses. Unlike other secreted factors, GPC4 contains the C-terminal GPI-anchorage signal. However, the mechanism by which membrane-tethered GPC4 is released from astrocytes is unknown. Using mouse primary astrocyte cultures and a quantitative luciferase-based release assay, we show that GPC4 is expressed on the astrocyte surface via a GPI-anchorage. Soluble GPC4 is robustly released from the astrocytes largely by proteolytic shedding and, to a lesser extent, by GPI-anchor cleavage, but not by vesicular release. Pharmacological, overexpression, and loss of function screens showed that ADAM9 in part mediates the release of GPC4 from astrocytes. The released GPC4 contains the heparan sulfate side chain, suggesting that these release mechanisms provide the active form that promotes synapse maturation and function. Overall, our studies identified the release

\section{Significance Statement}

Astrocyte-derived diffusible factors regulate synapse development and function. However, the regulatory mechanism underlying the release of astrocyte-derived factors is poorly understood. Noting that, unlike many other secreted factors, glypican-4/GPC4 is GPI-anchored, we characterized the release mechanism of GPI-anchored GPC4 from astrocytes and identified the releasing enzyme. Heparan sulfated GPC4 is robustly released from the astrocytes largely by proteolytic shedding. In particular, ADAM9 in part mediates the release of GPC4 from astrocytes. Our study provides an enzymatic mechanism for releasing GPC4 from astrocytes and will provide a novel opportunity to understand the regulatory mechanism of neuron-glia communication for synaptogenesis. 
mechanisms and the major releasing enzyme of GPC4 in astrocytes and will provide insights into understanding how astrocytes regulate synapse formation and maturation.

Key words: ADAM9; astrocyte; glypican-4; GPI anchor; heparan sulfate; synaptogenesis

\section{Introduction}

Surface shedding is a mechanism by which specific surface proteins are cleaved and an ectodomain is released into the extracellular space. The shedding or release of surface proteins have been found to play important roles in various biological functions (Lichtenthaler et al., 2018). Ectodomain shedding can act to stop activity of proteins at the membrane, such as removing cell surface receptors or cell adhesion proteins, as in the case of $\mathrm{N}$-cadherin (Reiss et al., 2005). Alternatively, shedding can release an active protein from the cell surface to act as a long range, diffusible signaling factor such as syndecan-1 in FGF-2 activation during wound healing (Kato et al., 1998). In this context, shedding can allow for temporally and spatially regulated release or activity of a biomolecule. In other cases, shedding acts as a molecular switch, changing the signaling function of the protein from one role while anchored to the surface membrane to another when shed. For example, membrane-anchored TNF- $\alpha$ can activate TNFR2 on direct cell to cell contact to activate antiinflammatory pathways, however when shed, TNF- $\alpha$ acts as a paracrine signal to drive proinflammatory TNFR1 signaling (Grell et al., 1995). In other cases, released proteins can change the signaling functions of related receptor complexes, such as when released soluble $\alpha$ CNTFR converts the LIF receptor complex and transduces CNTF signaling in the cells that are not normally responsive to CNTF (Davis et al., 1993).

The release of surface proteins also been shown to play important roles in synapse development (Lichtenthaler et al., 2018). For example, Nogo-66 receptor ectodomain shedding drives excitatory synapse formation in vivo (Sanz et al., 2018). Proteolytic shedding of cell adhesion molecules causes the structural and functional modifications of synapses (Bajor and Kaczmarek, 2013; Nagappan-Chettiar et al., 2017). Glypican-4/GPC4 has been identified as a cell surface protein sufficient to drive neuronal synaptogenesis in vitro (Allen et al., 2012). GPC4 is a GPI-anchored protein (Filmus et al., 2008) and highly expressed in astrocytes (Cahoy et al., 2008; Zhang et al., 2014). Notably, GPC4

Received February 17, 2021; accepted July 15, 2021; First published July 22, 2021.

The authors declare no competing financial interests.

Author contributions: K.H. and S.P. designed research; K.H. performed research; K.H. and S.P. contributed unpublished reagents/analytic tools; K.H. and S.P. analyzed data; K.H. and S.P. wrote the paper.

This work was supported by the National Institutes of Health Grant R01NS102444 (to S.P.).

Acknowledgements: We thank Joosang Park for maintaining the mouse colony and experimental assistance; Williams, Shepherd, Sundquist labs for materials, equipment, and advice; and University of Utah Core facilities.

Correspondence should be addressed to Sungjin Park at sungjin.park@ neuro.utah.edu.

https://doi.org/10.1523/ENEURO.0069-21.2021

Copyright $@ 2021$ Huang and Park

This is an open-access article distributed under the terms of the Creative Commons Attribution 4.0 International license, which permits unrestricted use, distribution and reproduction in any medium provided that the original work is properly attributed. expressed in astrocytes are released from the cell and the released GPC4 facilitates synapse maturation in vitro (Allen et al., 2012). While GPC4's downstream mechanism of action of signaling through RPTPs been characterized (FarhyTselnicker et al., 2017), little is known about how GPC4 is shed or released from astrocytes. A better understanding of the release mechanism of GPC4 offers insights into the synaptogenic role of astrocytes in neuronal development and could lead to novel therapeutic targets for synaptopathic disorders such as autism, schizophrenia, Down syndrome, and epilepsy (Dowling and Allen, 2018; Wilson and NewellLitwa, 2018).

GPI-anchored proteins can be released from the producing cells via multiple mechanisms, which include proteolytic shedding, GPI-anchor cleavage, and vesicular release (Muller, 2018). There are GPI-anchored proteins which are shed through proteolytic mechanisms such as metalloprotease-mediated shedding of Prion protein and UL16 binding proteins, MHC Class I-related molecules (Fernández-Messina et al., 2010; Linsenmeier et al., 2018). GPI-anchored proteins can also be shed through a lipase-based cleavage mechanism (Park et al., 2013; van Veen et al., 2017). In this context, unlike proteolytic shedding which releases an ectodomain fragment, an entire protein is released from the cell. GPI-anchored proteins have also been shown to associate with a vesicular membrane or lipid particle while becoming separated from the cell (Muller, 2018).

The mechanism by which GPC4 is released has implications to the role GPC4 plays in downstream signaling. GPC4 is posttranslationally modified with heparan sulfate side chains at residues near the $\mathrm{C}$ terminus of the protein. These heparan sulfate side chains are necessary for the known synaptogenic signaling functions of GPC4 (Allen et al., 2012; Condomitti et al., 2018). Thus, identification of the released form of GPC4 is critical to understand how astrocyte-derived GPC4 regulates synaptogenesis.

Here, we show that GPC4 is released from the cell surface of astrocytes in vitro. We observed that GPC4 is released predominantly via proteolytic shedding, and to a lesser extent by GPI-anchor cleavage, but not by vesicular release. The released GPC4 contains heparan sulfate attachment, suggesting that both release mechanisms preserve the synaptogenic activity of GPC4 in the extracellular space. We also identify ADAM9 as a proteolytic sheddase for GPC4. Overall, these observations show that astrocytes release heparan sulfate containing GPC4 by multiple mechanisms.

\section{Materials and Methods}

\section{Animals}

All animal care and experiments were conducted in accordance with $\mathrm{NIH}$ guidelines and the IACUC committee of the University of Utah (protocol no. 21-02004). C57BI6/ 
$\mathrm{J}$ mouse lines were maintained under the normal housing conditions with food and water available ad libitum and $12 / 12$ hour (h) light/dark cycle in a dedicated facility. All primary astrocyte cultures were generated using neonatal wild-type (WT) mice of either sex.

\section{Cell culture}

Heterologous cell experiments were conducted using HEK293T cells. Cells were grown in culture media: DMEM (Invitrogen 11965-092), 10\% FBS (Invitrogen 16140071), $1 \%$ sodium pyruvate (Invitrogen 11360070), 1\% penicillin/ streptomycin (Invitrogen 15140-122). For Biochemistry experiments, cells were seeded between 0.15 and 0.2 million cells/well in PEl coated 12-well plates. Plasmid constructs (pCAG nHA GPC4; see Table 1) were transfected with Fugene (Promega E2691)/opti-MEM mixture according to manufacturer protocols; $24 \mathrm{~h}$ after transfection, culture media is changed to serum-free DMEM for biochemical experimental conditions.

Astrocyte culture was generated from P2 mice of either sex. P2 mouse cortexes are dissected and incubated in a Papain solution for $30 \mathrm{~min}$ at $37^{\circ} \mathrm{C}$. The tissue is then washed with glial media [MEM (Mediatech 15-010-CV), 10\% horse serum (HyClone SH30074.03), 1\% penicillin/ streptomycin/glutamine (Invitrogen 10378-016)] before pipette dissociation and filtering through a $70 \mu \mathrm{m}$ strainer. Astrocytes are plated on a Poly-D lysine (Millipore A-003E)/collagen (Advanced BioMatrix 5005-100ML)-coated T75 flask (Fisher 07-202-000). Astrocytes are grown to confluency, with complete media changes every 3-4d. Once confluent, astrocytes are harvested with an $8 \mathrm{~min}$ TrypLE digestion and resuspended in glial media. Astrocyte cell suspension is nucleofected using the Lonza Nucleofector 4D system at 3- $\mu$ g plasmid DNA per construct (pZac2.1 Nluc-GPC4, pZac2.1 Nluc-GPC4 ${ }^{\mathrm{sec}}$, pZac2.1 Nluc-GPC4 ${ }^{\text {TM }}$, pZac2.1 Nluc-Prion, pSilencer 1.0 U6 shRNA; see Table 1), pCMV3 C-HA ADAM9 (Sino Biological MG50044-CY), pCS2 cHA3 mADAM10 (Park et al., 2013) with setting CL-133, before being plated on PolyD lysine/collagen-coated 12-well plates and allowed to grow to confluency for biochemical experiments. Once confluent, glial media is changed to DMEM high-glucose (Invitrogen 11960069) media for biochemical assays and experimental treatments.

\section{Cloning}

GPC4 was cloned from mouse cDNA library from RT$P C R$ and inserted into a pCAG vector (Table 1, set 1). An $\mathrm{HA}$ tag was inserted after GPC4's signal peptide sequence by long primer (Table 1, set 2). This construct, pCA N-HA GPC4, was used for HEK293T cell experiments. NlucGPC4 was developed by insertion of Nanoluciferase (Nluc; from Addgene plasmid \#66579) after the cleavage site of the GPC4 signal peptide (Table 1, set 3), then cloning the resulting construct into a pZac2.1 GFABC1D vector (Addgene 92281; Table 1, set 4). A secreted form of GPC4 (Nluc-GPC4 ${ }^{\mathrm{sec}}$ ) was generated by introducing a stop codon before the C-terminal GPI-anchorage signal. A transmembrane form of GPC4 (Nluc-GPC4 ${ }^{\mathrm{TM}}$ ) was generated by replacing the $\mathrm{C}$-terminal GPI anchorage signal with rat CD2 transmembrane protein (NM_012830.1). Two PCR amplicons including the GPC4 lacking the C-terminal GPIanchorage signal and CD2 antigen lacking the $\mathrm{N}$-terminal signal peptide were served as the PCR templates to produce chimeric DNA, which is further subcloned into pZac2.1 GFABC1D vector. Nluc-Prion was cloned from RT-PCR of mouse cDNA library and inserted into pZac2.1 GFABC1D vector (Table 1, set 5). shRNA constructs were designed in silico, and cloned into pSilencer 1.0 U6 with long primers (Table 1, sets 6,7 , and 8 for control, Adam9, and Adam10, respectively).

\section{Pharmacology}

Astrocytes were cultured as described above. Before treatment, the media of astrocytes were changed to serum-free media and incubated overnight. Astrocytes are then changed into fresh serum-free media with and without treatment (25 $\mu \mathrm{m}$ GM6001) and incubated for $3 \mathrm{~h}$ before collection and astrocytes are lysed for biochemical analysis or luciferase assay.

Phosphoinositide phospholipase C (PI-PLC) is a bacterial enzyme that robustly cleaves GPI-anchored proteins from the cell surface membrane. For biochemical assays, PI-PLC is added to cell culture media for $0.5-3 \mathrm{~h}$ for acute treatment or overnight for chronic treatment. Both time frames result in near complete release of all GPI-anchored proteins from the cell surface.

\section{Toxicity assay}

Cell death in primary astrocyte cultures was assayed using the Lonza ToxiLight Cytotoxicity BioAssay kit (Lonza LT17-217). Briefly, $20 \mu$ l of cultured media was collected, and mixed with $100-\mu l$ kit detection reagent. Samples were incubated for $5 \mathrm{~min}$, then luminescence was measured with a plate reader. Higher levels of luminescence are driven by the release of adenylate kinase from damaged cells, which react with ADP and luciferase in the assay buffer to produce bioluminescence. A toxic concentration of Edelfosine (50 $\mu \mathrm{m})$ was used as a positive control for cell death.

\section{Western botting}

Media samples were mixed with $4 \times$ gel loading buffer (200 mm Tris, 8\% SDS, 24\% glycerol, and 0.04\% bromophenol blue, $\mathrm{pH}$ 6.8), while lysate samples were created by lysing cells with $1 \times$ gel loading buffer and syringe lysing. Where necessary, astrocyte conditioned media (ACMs) samples were concentrated $20 \times$ in $10 \mathrm{kDa}$ cutoff centrifuge protein concentrators (Thermo Fisher Scientific 88517 ) to enrich for WT GPC4 before mixing with gel loading buffer. Samples were loaded into $10 \%$ gels and run at $140 \mathrm{~V}$ for $70 \mathrm{~min}$, then transferred at $100 \mathrm{~V}$ for $60 \mathrm{~min}$ on PVDF membranes. Membranes were blocked for $1 \mathrm{~h}$ with $5 \%$ milk in TBST (100 mm Tris, $150 \mathrm{~mm} \mathrm{NaCl}$, and $0.1 \%$ Tween 20, pH 7.5) before placed in primary antibody: HA (BioLegend 901501), GAPDH (Cell Signaling Technology 2118S), IGFBP2 (Cell Signaling Technology 3922S), Nluc (R\&D Biosystems MAB100261), and CD9 (Abcam ab92726). Blots were probed with HRP-conjugated secondaries 
Table 1: Primers used

\begin{tabular}{|c|c|c|c|c|}
\hline Set & FP/RP & Primer sequence & Gene name & Vector backbone \\
\hline \multirow[t]{2}{*}{ Set 1} & FP & CGATGGCCGGCCACCATGGCACGCTTAGGCTTGCTC & Gpc4 & pCAG GPC4 \\
\hline & $\mathrm{RP}$ & GCACTTCCGAGCAACTITC & & \\
\hline \multirow[t]{4}{*}{ Set 2} & $\mathrm{FP}$ & CCACCATGGCACGCTTAGGCTTGCTCGCGCTCCTCTGCACCCTGGCCG & Gpc4 (endogenous signal peptide) & pCAG GPC4 \\
\hline & & CGCTCAGCGCCTCGCTGCTGGCTGATATCGCGGAGCT & & \\
\hline & $\mathrm{RP}$ & CCGCGATATCAGCCAGCAGCGAGGCGCTGAGCGCGGCCAG & & \\
\hline & & GGTGCAGAGGAGCGCGAGCAAGCCTAAGCGTGCCATGGTGGCCGG & & \\
\hline \multirow[t]{3}{*}{ Set 3} & FP & TCGCTGCTGGCTGATATGGTCTTCACACTCGAAGATTTCG & Nano luciferase & pCAG GPC4 \\
\hline & $\mathrm{RP}$ & CTTGAGCTCCGCGATCTTATCGTCGTCATCCTTGTAAT & & \\
\hline & & CCGCCAGAATGCGTTCGCACAG & & \\
\hline \multirow[t]{2}{*}{ Set 4} & FP & ATAGGCTAGCCTCGAGACCATGGCACGCTTAGGCTTG & Nluc-GPC4 & pZac2.1 Nluc-GPC4 \\
\hline & $\mathrm{RP}$ & CCGGGTCGACTCTAGATTATCTCCACTCTCCCTGCAC & & \\
\hline Set $4 \mathrm{~A}$ & $\mathrm{RP}$ & ATCGTCTAGATCATTACTTGTACAGCTCGTCCAT & Nluc-GPC4 $4^{\mathrm{TM}}$ & \\
\hline \multirow[t]{2}{*}{ Set 5} & $\mathrm{FP}$ & ATTCTGGCGTCCGGAAAAAAGCGGCCAAAGCCTGGA & Prion & pZac2.1 Nluc-Prion \\
\hline & $\mathrm{RP}$ & TCCAGGCTTGGCCGCTITITCCGGACGCCAGAAT & & \\
\hline \multirow[t]{2}{*}{ Set 6} & FP & AATTCGCGCCTAGGTCCGAACCCCGTTCGGACCTAGGCGCGAATTATTTTT & shRNA control & pSilencer 1.0 U6 \\
\hline & $\mathrm{RP}$ & AATTAAATTCGCGCCTAGGTCCGAACGGGGTTCGGACCTAGGCGCGAATTGGCC & & \\
\hline \multirow[t]{2}{*}{ Set 7} & $\mathrm{FP}$ & ACAAGAACCACAATTACTAGATCAAGAGTCTAGTAATTGTGGTTCTTGTATTTTT & Adam9 shRNA1 & pSilencer 1.0 U6 \\
\hline & $\mathrm{RP}$ & AATTAAAAATACAAGAACCACAATTACTAGACTCTTGATCTAGTAATTGTGGTTCTTGTGGCC & & \\
\hline \multirow[t]{2}{*}{ Set 7A } & $\mathrm{FP}$ & GCCAATAACGTCTGCATTITCAAGAGAAATGCAGACGTTATTGGCATITTT & Adam9 shRNA2 & pSilencer 1.0 U6 \\
\hline & RP & AATTAAAAATGCCAATAACGTCTGCATTTTCAAGAGAAATGCAGACGTTATTGGCGGCC & & \\
\hline \multirow[t]{2}{*}{ Set 8} & FP & GCAAATGATACCCTTACAGTTTCAAGAGAACTGTAAGGGTATCATTTGCATTTTT & Adam10 shRNA1 & pSilencer 1.0 U6 \\
\hline & $\mathrm{RP}$ & AATTAAAAATGCAAATGATACCCTTACAGTTCTCTTGAAACTGTAAGGGTATCATTTGCGGCC & & \\
\hline \multirow[t]{2}{*}{ Set $8 \mathrm{~A}$} & $\mathrm{FP}$ & GGAAGACAGTTCAACCTACGATCAAGAGTCGTAGGTTGAACTGTCTTCCATTTTT & Adam10 shRNA2 & pSilencer 1.0 U6 \\
\hline & RP & AATTAAAAATGGAAGACAGTTCAACCTACGACTCTTGATCGTAGGTTGAACTGTCTCCGGCC & & \\
\hline \multirow[t]{2}{*}{ Set 9} & $\mathrm{FP}$ & GGGGTGTGTAACAGCAATAAG & ADAM9 & Sequencing primers \\
\hline & RP & CAAGGGGGACGATTAGGAAG & & \\
\hline \multirow[t]{2}{*}{ Set 10} & $\mathrm{FP}$ & AACAGGCTTATCGCTATG & ADAM10 & Sequencing primers \\
\hline & $\mathrm{RP}$ & СТGСTGCTGACTTCTAAT & & \\
\hline \multirow[t]{2}{*}{ Set 11} & $\mathrm{FP}$ & CTGGAGAAACCTGCCAAGTA & GAPDH & Sequencing primers \\
\hline & RP & AGTGGGAGTTGCTGTTGAAG & & \\
\hline
\end{tabular}

[mouse (Jackson ImmunoResearch 715-035-151), rabbit (Jackson ImmunoResearch 705-035-003), rat (Jackson ImmunoResearch 112-035-003)]. Blots were developed using a chemiluminescent substrate (Thermo Scientific 34580).

\section{Luciferase assay}

Promega Nanoluc Furimazine is diluted 1:50 in assay substrate:assay buffer, then diluted 1:4 in 1× PBS. Media samples are cleared by centrifugation and $20 \mu \mathrm{l}$ of sample are loaded in 96-well plate wells. Lysate samples are generated by treatment of $1 \times$ passive lysis buffer (Promega E1941) to 12-well plate cultures, and $20 \mu$ l of sample are loaded in 96-well plate wells. Luminescence of individual wells was read in a plate reader after injection of $100 \mu \mathrm{l}$ Furimazine. Average luminescence value is calculated and normalized to either pretreatment or lysate samples as a transfection control.

\section{Biotinylation}

Cells are cooled on ice, then washed with PBS ++ (PBS, $1 \mathrm{~mm} \mathrm{CaCl}_{2}$, and $0.5 \mathrm{~mm} \mathrm{MgCl}_{2}, \mathrm{pH}$ 7.4) before 10min incubation of Sulfo-NHS-SS-biotin (Invitrogen 21331; $1 \mathrm{mg} / \mathrm{ml}$ in PBS ++ ). Biotin washed off with PBS ++ , then quenched with cold glial media, then washed again in PBS before incubated in serum-free media for $5 \mathrm{~h}$ at $37^{\circ} \mathrm{C}$. Incubation media collected, then cleared by centrifugation; $300 \mu \mathrm{l}$ 1:1 slurry strepavidin beads (Invitrogen
29201) were prepared with a $1 \times$ RIPA ( $150 \mathrm{~mm} \mathrm{NaCl}, 1 \%$ P-40, $0.5 \% \mathrm{NaDOC}, 0.1 \%$ SDS, and $25 \mathrm{~mm}$ Tris) wash, two wash buffer rinses ( $40 \mathrm{~mm}$ Tris, $\mathrm{pH} 7.6$ ) before resuspended in wash buffer as 1:1 slurry; $40 \mu \mathrm{l} 1: 1$ slurry used per $900 \mu \mathrm{l}$ media sample and incubated together with rotation at $4^{\circ} \mathrm{C}$ for $1 \mathrm{~h}$. Beads are spun down, supernatant is collected for analysis, then beads are washed with $1 \times$ wash buffer. Bead fraction is resuspended in elution buffer ( $2 \mathrm{M} \mathrm{NaCl}$ and $40 \mathrm{~mm}$ Tris, $\mathrm{pH}$ ) and slurry is used for Western blot and Nluc assays.

\section{Ultracentrifugation}

Astrocytes are grown after nucleofection in T75 flasks until confluent. Astrocytes are incubated in serum-free media for $24 \mathrm{~h}$ before media is collected and subjected to serial centrifugations $(10 \mathrm{~min} 300 \times g, 10 \mathrm{~min} 2000 \times g$, 30 min $10,000 \times g)$. A $1.5 \mathrm{~h}, 100,000 \times g$ centrifugation pellets extra cellular vesicles (Konoshenko et al., 2018). The supernatant and pellet are collected for Nluc assays and Western blot analysis.

\section{Toxin pulldown}

$\alpha$-Toxin (AT) plasmid was generously provided by Yeongjin Hong at the Chonnam National University, Korea (Shin et al., 2006). BL21 cells (Fisher NC9122855) expressing $\alpha$-toxin are pelleted and resuspended in $0.5 \times$ RIPA (75 mm NaCl, 0.5\% P-40, 0.25\% NaDOC, 0.05\% SDS, and $12.5 \mathrm{~mm}$ Tris) before being sonicated. Cell 


\begin{tabular}{|c|c|c|c|}
\hline Data & $\begin{array}{l}\text { Data structure } \\
\text { (normality test) }\end{array}$ & Type of test & Power \\
\hline Figure $2 D$, lysate measurement of Nluc-GPC4 with and without PI-PLC treatment & Yes & Unpaired $t$ test & Cohen's $d=7.29, p<0.0001$ \\
\hline Figure $2 D$, media measurement of Nluc-GPC4 with and without PI-PLC treatment & Yes & Unpaired $t$ test & Cohen's $d=3.00, p<0.0001$ \\
\hline Figure $2 E$, overnight release of GPC4 compared with Prion, normalized to lysate & Yes & Unpaired $t$ test & Cohen's $d=3.59, p<0.0001$ \\
\hline Figure $3 A$, live surface Biotinylation of GPC4 release over time & Yes & $\begin{array}{l}\text { ANOVA; Tukey's multiple } \\
\text { comparisons }\end{array}$ & Cohen's $d=1.012, p<0.0001$ \\
\hline Figure $3 B$, ultracentrifugation of Nluc-GPC4 in ACMs & Yes & Unpaired $t$ test & Cohen's $d=7.829, p<0.0001$ \\
\hline Figure 3C, pulldown of Nluc-GPC4 by $\alpha$ Toxin & Yes & $\begin{array}{l}\text { ANOVA; Tukey's multiple } \\
\text { comparisons }\end{array}$ & Cohen's $d=7.659, p<0.0001$ \\
\hline Figure $4 A$, reduction of Nluc-GPC4 by protease inhibitor GM6001 & Yes & Unpaired $t$ test & Cohen's $d=1.118, p=0.0003$ \\
\hline Figure $4 B$, reduction of Nluc-GPC4 release by Adam 9 shRNA1 KD & Yes & $\begin{array}{l}\text { ANOVA; Tukey's multiple } \\
\text { comparisons }\end{array}$ & Cohen's $d=1.832, p<0.0001$ \\
\hline Figure $4 B$, reduction of Nluc-GPC4 release by Adam 9 shRNA2 KD & Yes & $\begin{array}{l}\text { ANOVA; Tukey's multiple } \\
\text { comparisons }\end{array}$ & Cohen's $d=2.63, p<0.0001$ \\
\hline Figure $4 C$, fold gene expression by qPCR for shRNA KD experiments Adam9 shRNA1 & Yes & One-sample $t$ test & Cohen's $d=1.393, p=0.0190$ \\
\hline Figure $4 C$, fold gene expression by qPCR for shRNA KD experiments Adam 9 shRNA2 & Yes & One-sample $t$ test & Cohen's $d=0.799, p=0.1076$ \\
\hline Figure $4 C$, fold gene expression by qPCR for shRNA KD experiments Adam 10 shRNA1 & Yes & One-sample $t$ test & Cohen's $d=10.33, p<0.0001$ \\
\hline Figure $4 C$, fold gene expression by qPCR for shRNA KD experiments Adam 10 shRNA2 & Yes & One-sample $t$ test & Cohen's $d=1.089, p=0.0444$ \\
\hline Figure $4 D$, induction of Nluc-GPC4 release by Adam9 Overexpression & Yes & $\begin{array}{l}\text { ANOVA; Tukey's multiple } \\
\text { comparisons }\end{array}$ & Cohen's $d=3.482, p<0.0001$ \\
\hline Figure $5 A$, heparinase treatment of Nluc-GPC4 vs Control & Yes & One-sample $t$ test & Cohen's $d=2.93, p=0.0365$ \\
\hline Figure 5B, pulldown of Nluc-GPC4 by $\alpha$ Toxin in PI-PLC control vs basal release conditions & Yes & Unpaired $t$ test & Cohen's $d=0.390, p=0.9467$ \\
\hline Extended Data Figure 1-1, cytotoxicity assay of PI-PLC treatment & Yes & $\begin{array}{l}\text { ANOVA; Tukey's multiple } \\
\text { comparisons }\end{array}$ & Cohen's $d=0.907, p=0.964$ \\
\hline Extended Data Figure 1-1, cytotoxicity assay of Edelfosine treatment & Yes & $\begin{array}{l}\text { ANOVA; Tukey's multiple } \\
\text { comparisons }\end{array}$ & Cohen's $d=2.870, p<0.0001$ \\
\hline Extended Data Figure 2-1, lysate measurement of Nluc-GPC4-TM with and without PI-PLC treatment & Yes & Unpaired $t$ test & Cohen's $d=0.49, p=0.19$ \\
\hline Extended Data Figure 2-1, media measurement of Nluc-GPC4 = TM with and without PI-PLC treatment & Yes & Unpaired $t$ test & Cohen's $d=0.50, p=0.27$ \\
\hline
\end{tabular}

extract is filtered and incubated with washed TALON beads (Takara 635501 ) at $4^{\circ} \mathrm{C}$ for $30 \mathrm{~min}$. After washing, AT-coated TALON beads are resuspended as $1: 1$ slurry in wash buffer (20 mM Tris, $500 \mathrm{~mm} \mathrm{NaCl}, \mathrm{pH} 7.9)$, and $60 \mu \mathrm{l}$ of slurry is added to $900 \mu \mathrm{l}$ of media samples and incubated for $1 \mathrm{~h}$ at $25^{\circ} \mathrm{C}$. Beads are pelleted by centrifugation, supernatant is collected, beads are washed with wash buffer before being resuspended as a 1:1 slurry in wash buffer and used in Nluc assays.

\section{DEAE pulldown}

DEAE-Sepharose beads (Sigma DFF100-50ML) are prepared with sequential high and low salt washes $(2 \mathrm{M}$ $\mathrm{NaCl}, 40 \mathrm{~mm}$ Tris, $\mathrm{pH} 8.0$, and $40 \mathrm{~mm}$, Tris $\mathrm{pH} 7.6$, respectively) before resuspended as a 1:1 slurry in wash buffer. Media containing Nluc-GPC4 was added to DEAE 1:1 slurry, and incubated at room temperature with rotation for $2 \mathrm{~h}$. Media-DEAE mixture is centrifuged and supernatant is collected while the DEAE pellet is washed once with $10 \times$ bed volume wash buffer before wash buffer is aspirated. Nluc-GPC4 is eluted from DEAE beads with high salt ( $40 \mathrm{~mm}$ Tris and $2 \mathrm{M} \mathrm{NaCl}, \mathrm{pH} 8$ ) at room temperature for $30 \mathrm{~min}$ with mild agitation. Eluted Nluc-GPC4 in elution buffer is collected and used for luciferase assay.

\section{Heparinase treatment}

Samples containing Nluc-GPC4 were treated with Heparinase II (NEB P0735S) and Heparinase III (NEB P0737S) overnight at $37^{\circ} \mathrm{C}$, then subject to DEAE pulldown described above, before luciferase activity was assayed. Untreated samples were similarly incubated at $37^{\circ} \mathrm{C}$ and used as controls for Heparinase treatment for the DEAE pulldown and luciferase assay.

\section{Quantitative PCR (qPCR)}

Astrocytes were harvested $4 \mathrm{~d}$ after shRNA nucleofection and subjected to RNA extraction using TRI Reagent Solution (Invitrogen, AM9738). $1 \mu \mathrm{g}$ of extracted RNA was used for the RT reaction using PrimeScript High Fidelity RT-PCR kit (Takara, R022A), according to the manufacturer(s) instruction. $0.5 \mu \mathrm{l}$ from the total $20 \mu \mathrm{l}$ cDNA eluent was mixed with each $0.22 \mu \mathrm{m}$ Adam9 primers (Table 1, set 9), Adam10 primers (Table 1, set 10), or Gapdh (Table 1, set 11) with $23 \mu \mathrm{l}$ SYBR Select Master Mix (Applied Biosystems, 4472908). The PCR was conducted with total 40 cycles $\left(20 \mathrm{~s}\right.$ at $95^{\circ} \mathrm{C}, 20 \mathrm{~s}$ at $58^{\circ} \mathrm{C}$, and $40 \mathrm{~s}$ at $72^{\circ} \mathrm{C}$ ), using a Life Technologies QuantStudio 12K Flex instrument at the Genomics Core Facility, a part of the Health Sciences Cores of the University of Utah. Adam mRNA levels in the shRNA nucleofection was normalized by that of Gapdh and was compared with shRNA control nucleofection conditions.

\section{Statistical analyses}

Statistics were performed using GraphPad Prism software version 9 (Table 2). Experiments with two groups were compared with unpaired $t$ tests and $95 \%$ confidence intervals (Cls) were calculated for group means and the difference between means. Experiments with three or more groups were compared with ANOVA and Tukey's 
multiple comparisons, and $95 \%$ Cls were calculated for group means and the differences between means. Onesample $t$ tests were used for Figures $4 C, 5 A$ to calculate the difference between control and experimental groups. All data are plotted as mean, with error bars denoting $95 \% \mathrm{Cls}$ and all individual data points plotted. For all experiments, the $n$ numbers shown refer to the number of replicates, while $N$ numbers refer to the number of biological replicates.

\section{Results}

\section{GPC4 is expressed on the astrocyte surface via a GPI- anchor.}

In silico analysis indicates that GPC4 contains the Nterminal signal peptide and $\mathrm{C}$-terminal hydrophobic patch and is predicted to be a GPI-anchored protein (Fig. 1A). To directly test whether GPC4 is GPI-anchored on the cell surface, we first examined whether GPC4 is released from the cell surface by the application of PI-PLC, a bacterial GPI-anchor cleaving enzyme (Chen et al., 1998). Nterminal HA-tagged GPC4 was transfected into HEK293T cells, and media were collected with and without PI-PLC treatment (Fig. 1B). PI-PLC treatment greatly facilitated the release of GPC4, indicating that GPC4 is GPI-anchored on the HEK293T cell surface. Interestingly, GPC4 was detected in the medium by Western botting without PI-PLC treatment, suggesting constitutive release of GPC4 from the HEK293T cells.

Next, we tested whether endogenous GPC4 expressed on the astrocytes is GPI-anchored. Primary astrocytes from mouse cortex were treated with or without PI-PLC overnight and collected ACMs was assayed for shed GPC4. Because of the poor antigenicity of GPC4, we concentrated the ACM for Western bottings (see Materials and Methods) Consistent with observations in heterologous cells, PI-PLC treatment on astrocytes greatly facilitated the shedding of endogenous GPC4 into the media (Fig. 1C). In contrast, PI-PLC treatment did not increase the secretion of IGFBP2 from astrocytes. Interestingly, PI-PLC treatment also slightly increased the level of thrombospondin 1 (TSP1) in the culture medium, an astrocyte secreted protein involved in synaptogenesis (Christopherson et al., 2005). This result suggests that a portion of secreted TSP1 is associated with GPI-anchored proteins on the astrocyte surface. TSP1 is known to bind surface heparan sulfate, and a portion of secreted TSP1 from astrocytes may be recruited on the astrocyte surface, possibly as a mechanism to control the diffusion of secreted TSP1 (Feitsma et al., 2000). To further test whether PI-PLC treatment non-specifically releases cellular contents from astrocytes, we performed a cell toxicity assay. Overnight treatment of astrocytes with PI-PLC did not affect the cell viability, while edelfosine treatment $(50 \mu \mathrm{m}$, $1 \mathrm{~h}$ ), which induces apoptosis by activating Fas/CD95 receptor and PLC inhibition (Mollinedo et al., 2004), caused astrocyte cell death (Extended Data Fig. 1-1).

To determine the degree of GPI-anchorage of GPC4 on the astrocyte cell surface, we performed a surface biotinylation assay, which allows for labeling of all surface proteins at the time of biotinylation. PI-PLC pretreatment
A

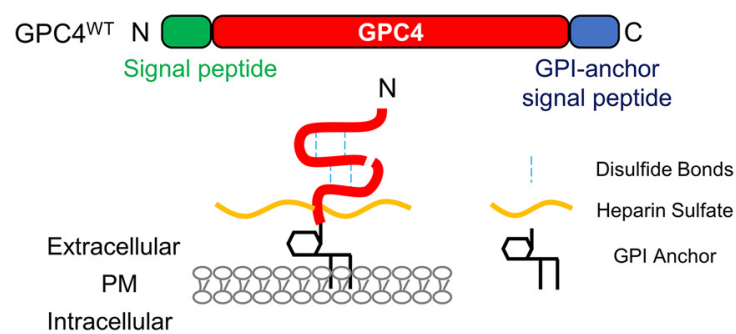

B

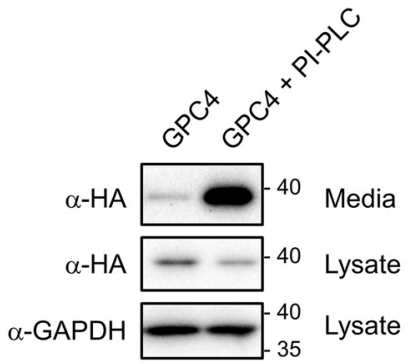

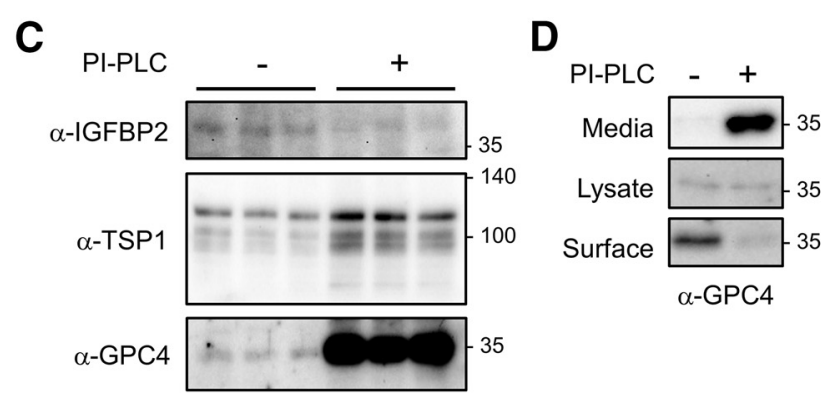

Figure 1. GPC4 is expressed on the astrocyte surface via a GPI-anchorage. A, Diagram of GPC4 sequence, protein, and posttranslational modifications. GPC4 contains an N-terminal signal peptide and GPI-anchor signal peptide for GPI-anchor attachment. Mature GPC4 is subject to furin cleavage, intrachain di-sulfide bonds, heparan sulfate attachment, and GPI-anchorage to the cell surface membrane. $\boldsymbol{B}$, Western blotting of HA tagged GPC4 construct expressed in HEK293T cells. The $\sim 37-$ $\mathrm{kDa}$ band shows the HA tagged N-terminal GPC4 in the reducing condition. PI-PLC treatment in HEK293T cells drives robust release of GPC4; however, GPC4 is detected in media in the absence of PI-PLC. $\boldsymbol{C}$, Western blotting of concentrated ACMs for endogenous GPC4, IGFBP2, and TSP1, with and without PIPLC treatment. PI-PLC treatment strongly facilitates release of endogenous GPC4 from astrocytes. $\boldsymbol{D}$, Biotinylation of surface GPC4 shows that pretreatment of PI-PLC removes endogenous GPC4 from the cell surface of astrocytes. Extended Data Figure 1-1 supports Figure 1.

removed the biotinylated GPC4 from the cell lysate (Fig. $1 D$ ). These results indicate that GPC4 is expressed on the astrocyte surface predominantly via GPI-anchor.

\section{The release of GPC4 from astrocytes}

Proteomic analysis of ACM showed that GPC4 is highly enriched in the astrocyte secretome, ranking 20th in 


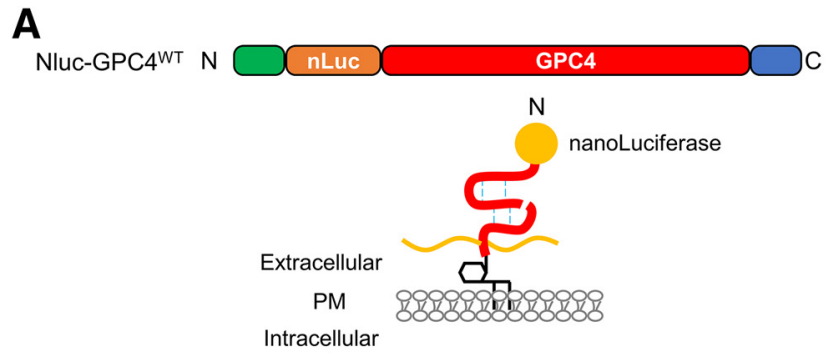

B

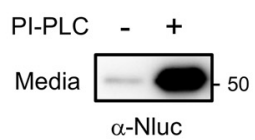

D

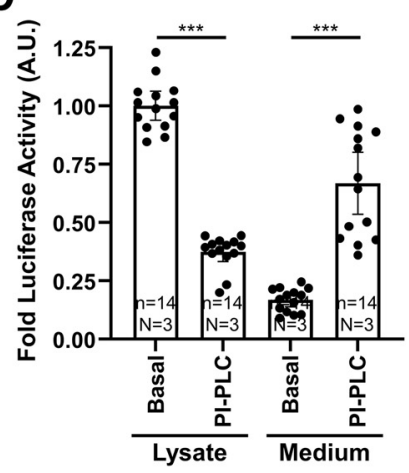

C

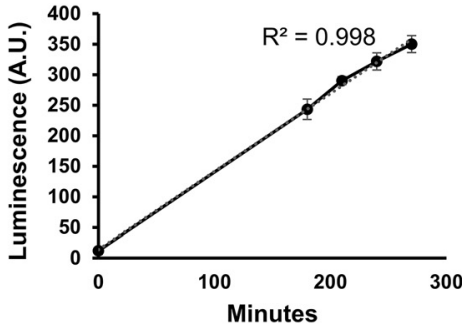

E

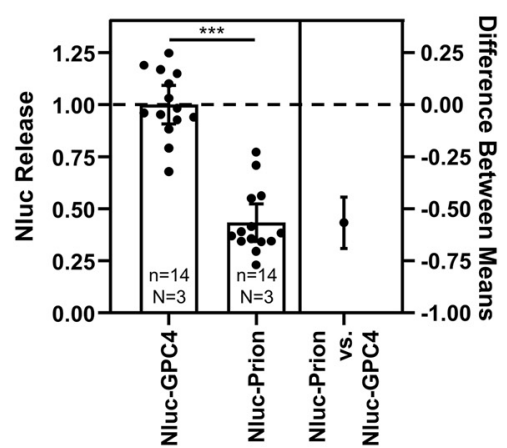

Figure 2. Luciferase assay for quantifying the release of GPC4 from astrocytes. $\boldsymbol{A}$, Nluc is inserted at the N terminus, after the endogenous signal peptide, to preserve GPC4 trafficking. $\boldsymbol{B}$, Primary astrocytes were nucleofected with Nluc-GPC4 and treated with and without PI-PLC. Western botting with $\alpha$-Nluc antibody showed the expected $20 \mathrm{kDa}$ size shift in the N-terminal fragment. PIPLC treatment facilitates the release of Nluc-GPC4, confirming the GPI-anchorage of the construct. C, Representative trace of one experiment showing the linear kinetics of GPC4 release from astrocyte culture $\left(R^{2}=0.998\right)$. Error bars indicate the standard error of the mean. $\boldsymbol{D}$, Astrocytes expressing Nluc-GPC4 were incubated in fresh media with and without PI-PLC for $3 \mathrm{~h}$, and Nluc signal was measured in the cell lysate and media. Nluc signal was normalized to untreated lysate conditions for each biological replicate. PIPLC treatment resulted in the decrease in the luciferase activity of the cell lysate and the corresponding increase in the activity in the media. These data show Nluc-GPC4 is quantitative in measuring released versus surface pools of GPC4. Error bars indicate $95 \% \mathrm{Cl}$ of the mean here and in following graphs. The requirement of the GPI-anchorage for PI-PLC-dependent release of GPC4 is shown in Extended Data Figure 2-1. E, The release rate (media over lysate activity) of Nluc-GPC4 and Nluc-Prion was normalized to Nluc-GPC4 release. Nluc-GPC4 is released $\sim 2$-fold more than Nluc-Prion ( $t$ test $p<0.0001$, Cohen's $d=3.59$ ); ${ }^{\star \star \star} p<0.001$.

Greco and colleagues, and ranking 28th in our mass spec analysis (data not shown; Greco et al., 2010). However, GPC4 undergoes extensive posttranslational modifications, including GPI-anchorage, convertase-mediated cleavage, disulfide-linkage, glycosylation, and heparan sulfate attachment (Fig. 1A; Filmus et al., 2008), which may cause the poor antigenicity. To quantify release kinetics of GPC4 from astrocytes, we generated a Nluc fusion protein construct after the endogenous signal peptide of GPC4 (Nluc-GPC4; Fig. 2A). Nluc is a small $(20 \mathrm{kDa})$ and bright luciferase reacting with a specific substrate, Furimazine (FMZ; Heise et al., 2013). Attaching the signal peptide to the $\mathrm{N}$ terminus of Nluc efficiently delivers the fusion protein into the secretory pathway. The secreted Nluc shows a linear lumine- scence in a wide range of Nluc concentration in the medium, which is suitable for quantifying the release of the membrane-tethered protein.

We examined the processing, trafficking, and kinetics of the construct as well as the posttranslational modifications important for GPC4 function. We nucleofected the Nluc-GPC4 construct under an astrocyte-specific GFABC1D promoter into astrocytes and measured the release of Nluc-GPC4 into the medium. Western botting with Nluc antibody showed the expected $\sim 20 \mathrm{kDa}$ shift of N-terminal fragment of GPC4 (Fig. 2B). Further, the treatment of PI-PLC robustly releases Nluc-GPC4 into the medium, indicating that N-terminal fusion of Nluc does not affect the GPI anchorage and surface expression of GPC4. It is also possible that PI-PLC treatment 
indirectly regulates the release of other types of proteins. For example, release of RECK, a GPI-anchored metalloprotease inhibitor from the cell surface can de-repress the surface sheddase, which in turn facilitates the shedding of non-GPI anchored proteins (Park et al., 2013). To test whether the release of Nluc-GPC4 by PI-PLC requires the GPI-anchorage, we generated a transmembrane form of GPC4 by replacing the C-terminal GPIanchorage signal with a rat CD2 transmembrane protein (Nluc-GPC4 ${ }^{\text {TM }}$ ). PI-PLC treatment did not affect the release of Nluc-GPC4 ${ }^{\mathrm{TM}}$ in astrocytes (Extended Data Fig. 2-1). This result further supports that PI-PLC treatment does not cause toxicity nor non-specific release of cellular proteins. Interestingly we observed linear accumulation of Nluc-GPC4 signal into the medium at the naive state $\left(R^{2}=0.998\right)$, further demonstrating the quantitative nature of the Nluc-GPC4 (Fig. 2C). Next, we measured the release rate of GPC4 per surface-tethered pool of GPC4. The amount of the surface pool of GPC4 is calculated by the reduction of Nluc-GPC4 signal in the cell lysate and the corresponding increase in the medium signal on PI-PLC treatment (Fig. 2D). We observed that $27 \%$ of the surface GPC4 is released into the medium over $3 \mathrm{~h}$, which indicates that $\sim 9 \%$ of the surface-expressed pool is released per hour at the naive state.

Noting the high release rate of GPC4, we compared the release rate of Prion, another GPI-anchored protein abundantly expressed in astrocytes (Cahoy et al., 2008; Zhang et al., 2014). The release rate (luminescence of the medium normalized to that of the cell lysate) of Nluc-GPC4 is $>2$-fold higher than that of Nluc-Prion, suggesting that even in this overexpression system, specific release mechanisms exist for different GPI anchored proteins and feature differential release kinetics (mean difference 95\% $\mathrm{Cl}=-0.7588$ to -0.4107 , Cohen's $d=3.92, p<0.0001$; Fig. 2E). Overall, Nluc-GPC4 retains posttranslational modifications of endogenous GPC4, including furin cleavage, and GPI-anchorage, and allows for rapid, non-destructive measurement of protein release from the cell surface into the media.

\section{GPC4 is released from the astrocyte cell surface}

How is GPI-anchored GPC4 is released from astrocytes? GPI-anchored proteins can be released from the cell via multiple mechanisms, which include (1) secretory pathway bypassing surface expression because of cleavage during processing in the secretory pathway, e.g., by GPLD1 (Hettmann et al., 2003; Park et al., 2013); (2) vesicular releases such as in exosomes or lipid associated particles (Muller, 2018; Dobrowolski et al., 2020); (3) GPIanchor cleavage on the cell surface (Park et al., 2013; van Veen et al., 2017); and (4) proteolytic shedding. Thus, we tested each possibility.

We first tested whether extracellular GPC4 at the basal state is shed from the cell surface or directly secreted without being retained on the astrocyte surface membrane. To test this possibility, we conducted a live surface biotinylation assay wherein astrocytes cell surface proteins were biotinylated before being allowed to recover and incubate at $37^{\circ} \mathrm{C}$ for $5 \mathrm{~h}$. Incubation media was then collected and assayed for biotinylated GPC4. If GPC4 is secreted directly from secretory pathways, the majority of collected GPC4 should not be biotinylated. Instead, we found that the ratio of biotinylated over total released GPC4 is similar between the basal state and the PI-PLC condition, which selectively releases the surface-bound pool (Fig. 3A). In contrast, a secreted form of Nluc-GPC4 $\left(\mathrm{GPC} 4^{\mathrm{sec}}\right)$, which lacks the C-terminal GPI-anchorage signal, is secreted without being biotinylated. While we expected the PI-PLC treatment condition to result in a maximal biotinylation ratio, the biotinylation ratio of the basal release was slightly higher than that of PI-PLC conditions (mean difference between basal vs PI-PLC 95\% Cl $=-0.3189$ to -0.1594 , Cohen's $d=1.012, p=0.0079$ ). PIPLC treatment rapidly removes all surface GPC4, which may facilitate the surface incorporation of newly synthesized, un-biotinylated GPC4. This would increase the release of un-biotinylated GPC4 by PI-PLC during the 5-h incubation as compared with untreated control. Together, these data demonstrate that GPC4 is released from the astrocyte surface membrane and not directly from a secretory pathway (Fig. 3A).

\section{GPC4 is not released through an extracellular vesicle mechanism}

Several GPI-anchored proteins are enriched in the extracellular vesicles (Vidal, 2020). These vesicular GPIanchored proteins retain the lipid tail and are associated with membrane structure. We tested for the possibility that GPC4 is released through an extra vesicular fraction like some GPI anchored proteins such as CNTFR (Choi et al., 2021). In this model of release, GPI-anchored GPC4 is expressed on the cell surface, and released while still bound to the membranes of extracellular vesicles either through exosomal or ectosomal pathways. To test this, media from astrocytes expressing Nluc-GPC4 were sequentially centrifuged, first to separate large particles such as floating cells and cell debris, then to isolate the vesicular fraction by ultracentrifugation (Konoshenko et al., 2018). We measured luciferase activity in the supernatant and pellet fractions, which correspond to soluble protein and vesicle associated fractions, respectively, and normalized the measurements to luciferase activity in the input sample. Luciferase activity shows no change between input and supernatant fractions, with no detection in the vesicular pellet fraction. To validate the ultracentrifugation assay, we tested the pellet fraction and confirmed enrichment of exosomal protein CD9 (Fig. 3B). Together, these data show minimal GPC4 presence in vesicular fractions, suggesting that extracellular vesicles are not the major route of GPC4 release in astrocytes.

\section{GPC4 is partially released through a GPI-anchor lipase}

A GPI-anchor lipase such as GDE family enzymes and GPLD1, cleaves the GPI anchor via PLC and PLD mechanism, respectively, and leaves the sugar core attached to the $\mathrm{C}$ terminus of the released protein. In contrast, a proteolytic cleavage would release a GPC4 ectodomain 
A

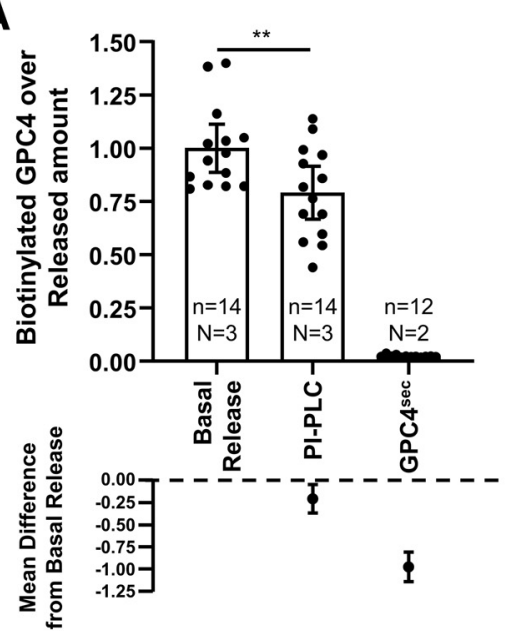

C

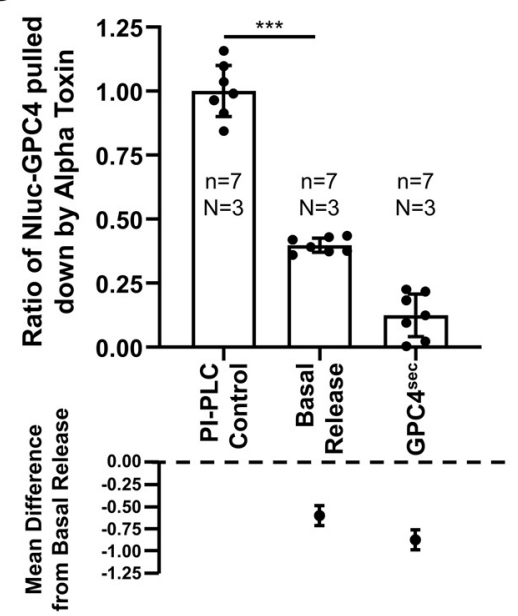

B
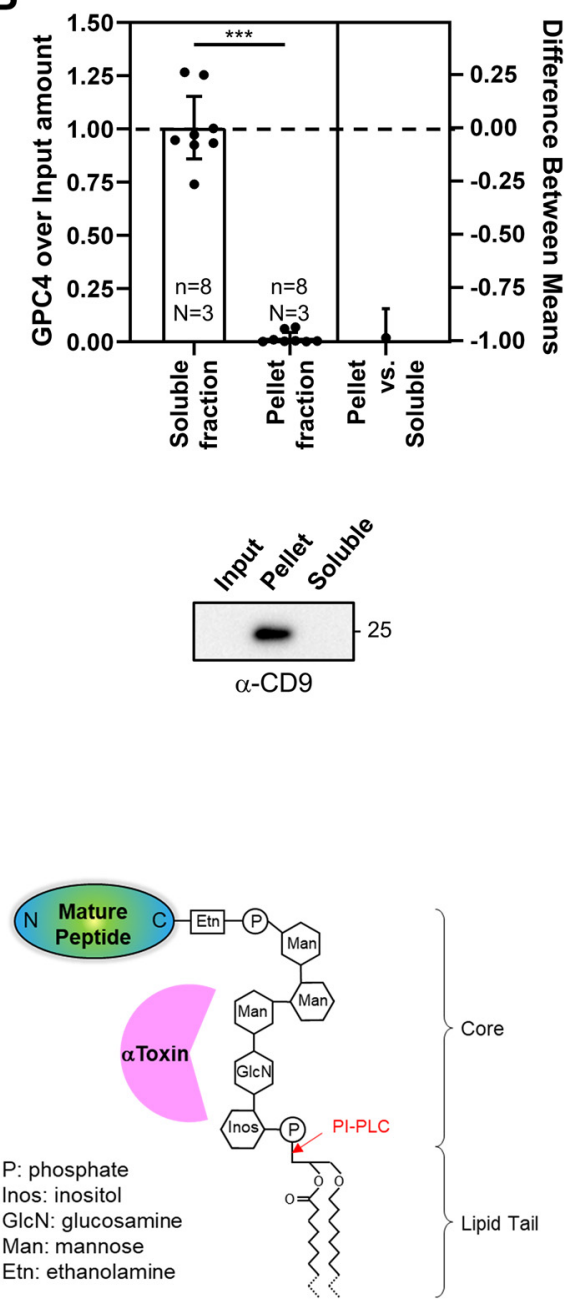

Figure 3. Characterizing the release mechanism of GPC4 using Nluc-GPC4. A, After biotinylating the surface proteins of astrocytes expressing Nluc-GPC4, cells were further incubated in the fresh medium for $5 \mathrm{~h}$. The released Nluc-GPC4 is collected from the medium, and the ratio of biotinylation of the released Nluc-GPC4 was measured (luciferase activity of avidin pulldown over medium input). As a control for the release from the cell surface, PI-PLC were treated during the incubation time. Both basal and PI-PLC induced GPC4 release occur from the cell surface. GPC ${ }^{\mathrm{sec}}$, which does not contain the GPI-anchor signal peptide and is thus not GPI-anchored, is directly secreted without membrane attachment and lacks biotinylation (basal vs PI-PLC ANOVA $p=0.0079$, Cohen's $d=1.012$ ). $\boldsymbol{B}$, Ultracentrifugation of ACMs containing Nluc-GPC4 was used to test for GPC4 association with extracellular vesicles as a potential release mechanism. Supernatant (soluble) fraction and pellet (vesicular) fraction Nluc were normalized to input Nluc luminescence. Nluc-GPC4 did not associate with the pellet (vesicular) fraction. An exosome marker, CD9, was enriched in the pellet (vesicular) fraction ( $t$ test $p<0.0001$, Cohen's $d=7.829$ ). $\boldsymbol{C}$, alpha toxin (AT), which binds to the glycan core of GPI-anchors, was used to pull down GPI-anchored proteins released through lipase activity. PI-PLC, lipase released Nluc-GPC4 is used as a positive control, while GPC4 ${ }^{\mathrm{sec}}$, lacking a GPI anchor, is used as a negative control. Nluc-GPC4 pulled down by AT is normalized to input Nluc-GPC4 measurements. Basal release shows partial binding to AT, indicating that $\sim 30 \%$ of Nluc-GPC4 is released through a lipase mechanism (PI-PLC vs basal release ANOVA $p<0.0001$, Cohen's $d=7.659$ ); ${ }^{* \star} p<0.01,{ }^{\star \star *} p<0.001$.

fragment that lacks the GPI-anchor. Noting that AT, a bacterial GPI-anchor binding protein, recognizes the sugar motif of the GPI-anchor (Hong et al., 2002; Shin et al., 2006), we developed a pulldown assay that isolates the species containing the core domain of the GPI-anchor among the released pool (Fig. $3 C$ ). We expressed AT-His in Escherichia coli and purified it with metal resins. To test between these possibilities, we first assayed the percentage of released GPC4 with GPI-anchor residues using a pulldown experiment. Surprisingly, we found that in comparison to our positive PI-PLC control, which releases the surface GPC4 through GPI-anchor lipase activity, constitutively released GPC4 is only partially captured by ATcoated beads (mean difference between PI-PLC control vs basal release $95 \% \mathrm{Cl}=-0.4897$ to -0.7146 , Cohen's $d=7.659, p<0.0001$; Fig. $3 C$ ). This suggests that while some GPC4 is released through lipase activity of the GPI anchor, a majority of GPC4 is released through a 
mechanism that removes the GPI anchor from the released form.

\section{GPC4 is partially released through a proteolytic mechanism}

The results of the AT pulldown immediately suggest proteolytic cleavage as the primary mechanism by which GPC4 is released from the astrocyte cell surface. We first examined the effect of protease inhibitors on GPC4 release. In line with our model of proteolytic cleavage, we observed $\sim 30 \%$ reduction in GPC4 release after treatment with a metalloprotease inhibitor GM6001, an inhibitor of $\mathrm{Zn}$ catalyzed proteases, including MMP and ADAM family proteases (mean difference $95 \% \mathrm{Cl}=-0.5445$ to -0.1723 , Cohen's $d=1.118, p=0.0003$; Fig. $4 A$; Webber et al., 2002).

\section{Determining the protease responsible for GPC4 shedding}

GM6001 treatment significantly blocked the basal release of GPC4, indicating that Zn-dependent MMPs and ADAM family proteases play a role in GPC4 shedding (Fig. $4 A$ ). To identify the protease that mediates GPC4 cleavage, we performed a gain of function screen with tissue inhibitors of metalloproteinases (TIMPs) which inhibit MMP and ADAM proteases (Brew and Nagase, 2010). Overexpression of TIMP 1 and TIMP 3 , which collectively inhibit MMPs and ADAMs 10, 12, 17, 28, and 33, was unable to reduce release of GPC4 from astrocytes (data not shown). These results suggest that GPC4 shedding may be mediated by other proteases that are not inhibited by TIMPs, which include ADAMs 8, 9, and 19 (Brew and Nagase, 2010).

In parallel, we performed shRNA screens of ADAM proteases (Adamts1, Adamts5, Adam9, Adam10, Adam17) that are abundantly expressed in astrocytes (data not shown; Li et al., 2019). Among ADAM family proteases we tested, we observed that shRNAs against Adam9 significantly reduced the release of GPC4 (Adam9 shRNA1 mean difference $95 \% \mathrm{Cl}=-0.34$ to -0.14 , Cohen's $d=1.832$, Tukey's $p=0.0019$; Adam9 shRNA2 mean difference $95 \% \mathrm{Cl}=-0.33$ to -0.19 , Cohen's $d=2.68$, Tukey's $p=0.0010$; Fig. 4B). Consistent with our observations of TIMP overexpression, Adam10 shRNA produced little effect on the release of GPC4 from astrocytes (Adam10 shRNA1 mean difference 95\% Cl $=0.172$ to -0.080 , Cohen's $d=0.291$, Tukey's $p=0.8999$; Adam 10 shRNA2 mean difference $95 \% \mathrm{Cl}=0.150$ to -0.063 , Cohen's $d=0.315$, Tukey's $p=0.8999$; Fig. $4 B$ ).

qRT-PCR of wells transfected with a shRNA construct showed knock-down efficiency ranging from 0.1 - to 0.5 fold except in one biological replication of Adam 9 shRNA2 (Fig. 4C). Incomplete knock-down may cause mild effect of Adam9 knock-down on GPC4 release. Interestingly, reductions in Nluc-GPC4 release are consistently seen in Adam 9 shRNAs and not by Adam10 shRNAs. Since double nucleofection rate is near complete within the transfected cells and the release rate of Nluc-GPC4 is insensitive to the transfection rate after normalizing the luminescence in the lysate (data not shown), knock-down of Adam9 within the same cells expressing Nluc-GPC4 may produce more consistent effects on Nluc-GPC4 release than overall reduction of the mRNA level of the culture. Taken together, these results indicate that ADAM9 in part mediates the release of NlucGPC4.

To test whether ADAM9 is sufficient to induce the release of GPC4 from astrocytes, we overexpressed ADAM9 together with the Nluc-GPC4 reporter. Our results show that overexpression of ADAM9 and not ADAM10 facilitated GPC4 release from astrocytes (ADAM9 mean difference $95 \% \mathrm{Cl}=-5.606$ to -4.007 , Cohen's $d=3.482, p<0.0001$; ADAM10 mean difference $95 \% \mathrm{Cl}=-0.8448-0.7549$, Cohen's $d=0.1542$, $p=0.99$; Fig. 4D). Taken together, these data show that ADAM9 or its downstream effectors facilitate the release of GPC4 from astrocytes.

\section{Constitutively shed GPC4 predominantly contains the heparan sulfate side chains}

Heparan sulfate attachment to GPC4 plays a critical role in downstream signaling, including known synaptogenic functions through RPTPs and GPR158 (Condomitti et al., 2018; Farhy-Tselnicker et al., 2017). Unlike GPI-anchor cleavage, which releases full-length protein, proteolytic shedding could generate multiple extracellular fragments. Thus, it is critical to determine whether the released GPC4 contains heparan sulfate attachment sites after proteolytic shedding. To test heparan sulfate attachment to released GPC4, we performed a pulldown assay with an anion exchange column, which binds to the negative charge of heparan sulfate chain (Kojima et al., 1992). As a positive control, we tested whether the surface GPC4 contains heparan sulfate attachment. Treatment of basal condition medium with heparanases reduced the binding of nluc-GPC4 to the column (mean difference $95 \% \mathrm{Cl}=-1.091$ to -0.3206 , Cohen's $d=4.153$, onesample $t$ test $p=0.0365$; Fig. $5 A$ ), indicating that DEAE binding of GPC4 requires heparan sulfate attachment. Interestingly, the released GPC4 in the basal condition shows a similar binding affinity as that acutely released from the cell surface by PI-PLC (mean difference $95 \% \mathrm{Cl}$ $=-0.2504-0.2181$, Cohen's $d=0.3909, p=0.9467$; Fig. $5 B)$. These results show that constitutively shed NlucGPC4 from astrocytes contains the heparan sulfate modification, indicating that the cleavage site of GPC4 is located downstream of heparan sulfate attachment sites. Overall, the released GPC4 from astrocytes by either mode contains the heparan sulfate posttranslational modification, which is required for its downstream signaling for synapse development.

\section{Discussion}

Here, we show that GPC4 is expressed on the surface membrane via GPI-anchorage in astrocytes. Our results show that GPC4 is robustly released from the astrocyte surface predominantly by a proteolytic and partially by a lipase shedding mechanism. Soluble GPC4 released by either mode contains the heparan sulfate post- 
A

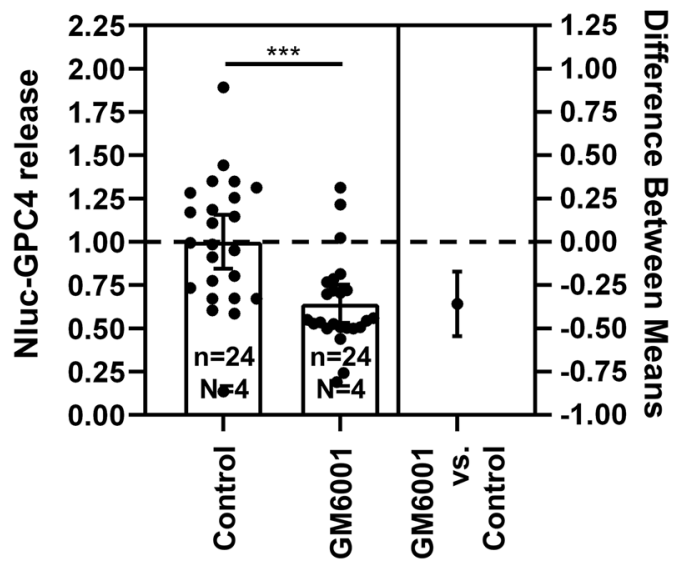

C

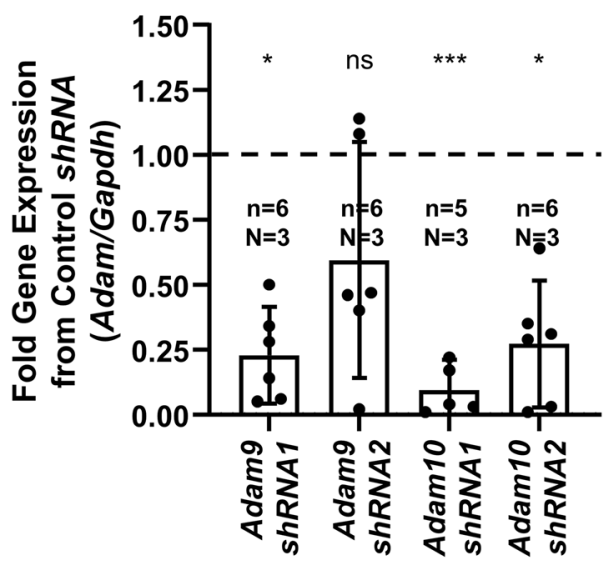

B

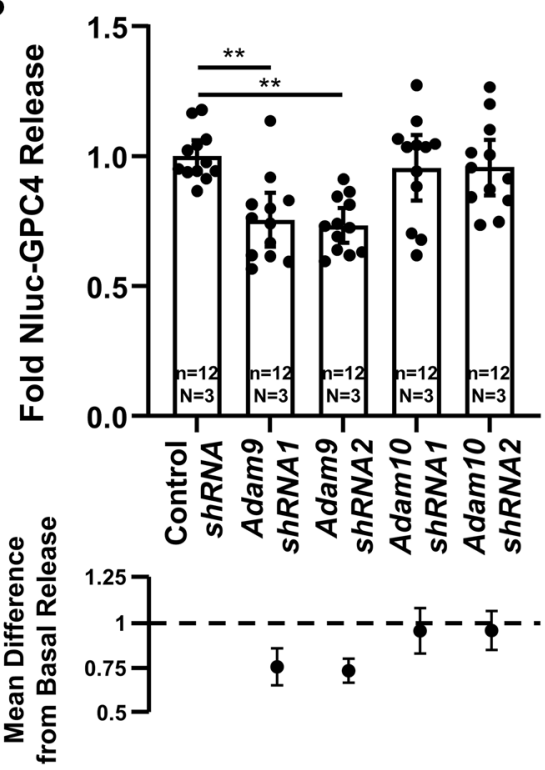

D

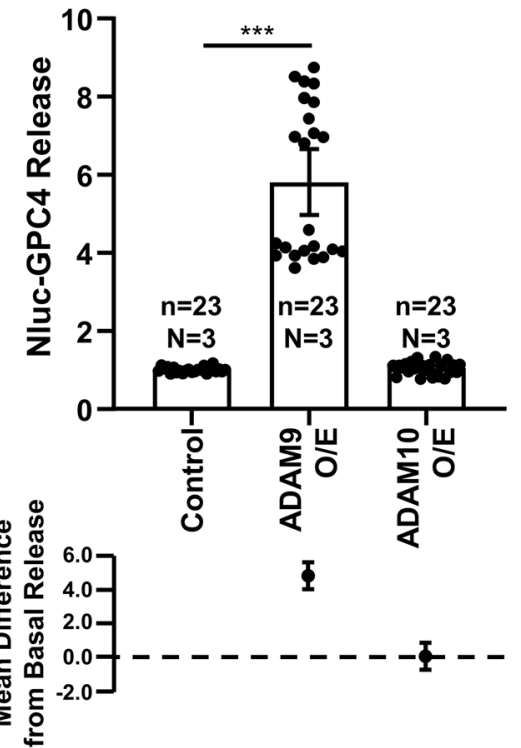

Figure 4. ADAM9 mediates the release of GPC4 from astrocytes. $\boldsymbol{A}$, GM6001 (25 $\mu \mathrm{M})$, an inhibitor of $\mathrm{Zn}^{2+}$-based proteases including the MMP and ADAM family proteases, reduced the release of GPC4 ( $t$ test $p=0.0003$, Cohen's $d=1.118$ ). B, shRNA knockdown of Adam9 and not Adam10 reduces the release of Nluc-GPC4 in astrocytes (control vs Adam9 shRNA1 Tukey's $p=0.0019$, Cohen's $d=1.832$; control vs Adam9 shRNA2 Tukey's $p=0.0010$, Cohen's $d=2.63$; control vs Adam10 shRNA1 Tukey's $p=0.8999$, Cohen's $d=0.291$; control vs Adam9 shRNA2 Tukey's $p=0.8999$, Cohen's $d=0.315)$. C, qPCR data of astrocyte Adam9 and Adam 10 knock-down replicates. $\Delta$-CT between Adam and Gapdh and $\Delta \Delta$-CT between control and target shRNA conditions were used to determine the fold change in expression. One biological replicate for Adam9 shRNA2 did not show reductions in Adam9 gene expression. Adam9 shRNA1 one-sample $t$ test $p=0.0190$, Cohen's $d=1.393$; Adam9 shRNA2 $p=0.1076$, Cohen's $d=0.799$; Adam10 shRNA1 $p<0.0001$, Cohen's $d=10.338$; Adam10 shRNA2 $p=0.0444$, Cohen's $d=1.089$. D, ADAM9 overexpression, and not ADAM10 overexpression (O/E), is capable of inducing the release of Nluc-GPC4 from astrocytes (control vs ADAM9 O/E ANOVA $p<0.0001$, Cohen's $d=3.482)$; ${ }^{*} p<0.05$, ${ }^{* *} p<0.01$, ${ }^{* *} p<0.001$, ns: not significant.

translational modification, which is necessary for GPC4 synaptogenic function. Further, our data identify ADAM9 as a protease that releases GPC4 from astrocytes.

GPC4 was identified as an astrocyte-derived signaling factor. The released GPC4 was shown to induce synapse formation and maturation in cultured neurons (Allen et al., 2012). Further study of astrocyte secreted GPC4 function identified a heparan sulfate-dependent interaction between GPC 4 and RPTP $\sigma$ and RPTP $\delta$ to drive neuronal pentraxin 1 (NP1)-mediated recruitment of AMPA receptors (Farhy-Tselnicker et al., 2017). GPC4 is also expressed in neurons and presynaptic GPC4 has been 
A

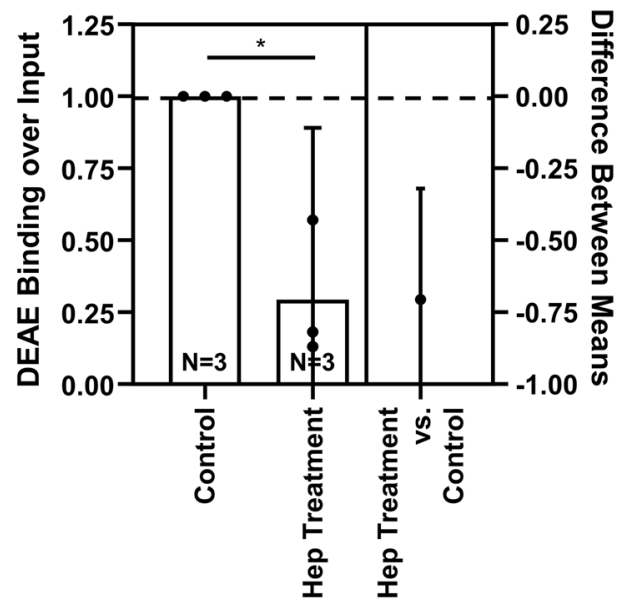

B

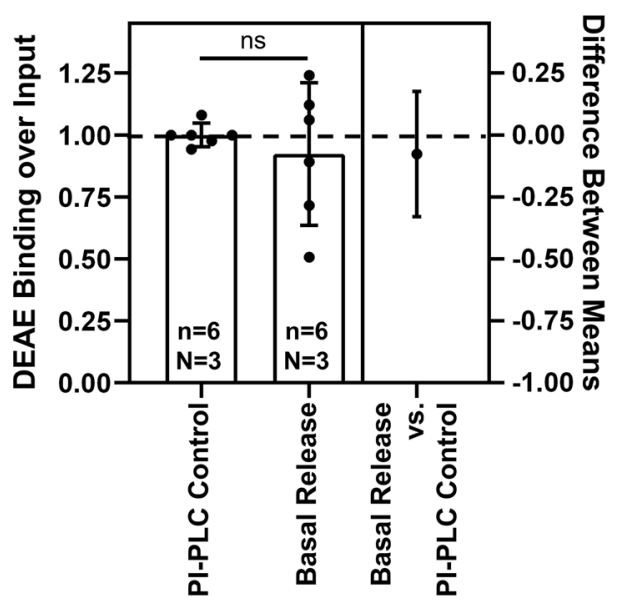

Figure 5. The released Nluc-GPC4 includes the heparan sulfate side chain. $\boldsymbol{A}$, Heparinase II and III treatment of media Nluc-GPC4 reduced the binding of GPC4 to DEAE anion exchange column, by removing the negatively charged heparan sulfate side chains (one-sample $t$ test $p=0.0365$, Cohen's $d=3.482$ ). $\boldsymbol{B}$, The surface Nluc-GPC4 released by PI-PLC showed similar binding to DEAE as basal release GPC4, indicating that both lipase and protease release mechanisms contain the heparan sulfate modification and are capable of known synaptogenic signaling functions ( $t$ test $p=0.9467$, Cohen's $d=0.390$ ); ${ }^{*} p<0.05$, ns: not significant.

found to play a role in the construction of mossy fiber synapses in the hippocampus via postsynaptic GPR158 (Condomitti et al., 2018). Given the observed roles of GPC4 in both a secreted form in astrocytes and in a cell membrane-tethered form in neurons, the release of GPC4 may play a distinct role in synaptogenesis depending on the cell type. For example, the release of GPC4 from astrocytes may facilitate the maturation of nearby synapses in a non-cell autonomous manner. Alternatively, release of GPC4 from the presynaptic membrane may inhibit the synaptic adhesion of a specific type of synapse, such as hippocampal mossy fiber synapses. Thus, elucidating the regulatory mechanism of surface GPC4 is critical to understand the physiological roles of GPC4 in synapse development.

To study the mechanism of GPC4 shedding, we found that it was necessary to develop a new tool to counter the poor antigenicity of GPC4. We developed a Nluc-based GPC4 release assay, which results in a quantitative, sensitive, and non-destructive assay capable of examining the temporal dynamics of GPC4 release. By using this method, we are able to distinguish the different modes of GPC4 release.

Biotinylation assays showed that GPC4 is released from the cell surface, not from the internal pool in the secretory pathway. It is notable that several GPI-anchored proteins are released with a lipid tail-intact form via lipidassociated particles such as on an extracellular vesicle or exosome (Muller, 2018). For example, exosomal GPC1 level is elevated in the serum of patients with pancreatic cancer and is associated with poor prognosis (Melo et al., 2015; Zhou et al., 2018). However, our results show that vesicular release is not a major route of GPC4 release from astrocytes and the majority of released GPC4 exists as a soluble form.

The soluble released form of GPC4 is generated largely by proteolytic shedding, which is partially blocked by
GM6001, a pan-metalloprotease inhibitor. Our TIMP overexpression and shRNA screens indicated that ADAM9 in part mediates the shedding of GPC4 in astrocytes. However, the magnitude of reduction is limited to 20$30 \%$, which suggests that there are other proteases working in parallel. Based on our GM6001 results, we expect these proteases to be unaffected by zinc-dependent proteolytic mechanisms, such as serine proteases like the (TMPRSS) family, which are not inhibited by GM6001 (Lam et al., 2015).

Our data show that ADAM9 overexpression is capable of shedding GPC4 from astrocytes. It is possible that ADAM9 induces GPC4 shedding through action on intermediate substrates or activation of an intermediate protease. For example, ADAM9 plays roles in APP shedding through regulation of surface expression and activity of ADAM 10 by acting as an ADAM10 sheddase (Moss et al., 2011). We specifically examined the ability of ADAM10 to shed GPC4, and found that neither overexpression nor shRNA knock-down of Adam10 is sufficient to impact GPC4 shedding in astrocytes. Based on these results, we favor the interpretation that GPC4 is a direct substrate of ADAM9 but do not rule out intermediate actors in this shedding mechanism.

AT pulldown assays showed that $\sim 30 \%$ of soluble GPC4 in the medium contains the core structure of the GPI-anchor, indicating a GPI lipase activity on GPC4 in astrocytes. There are several families of GPI-anchor Iipases that releases $\mathrm{GPI}$-anchored proteins. The glycerophosphodiester phosphodiesterase (GDPD) family and GPLD1 catalyze phospholipase-C (PLC) and PLD cleavage of the GPI-anchor, respectively (Hettmann et al., 2003; Park et al., 2013; van Veen et al., 2017). Overexpression of GDE2/GDPD5 in neuroblastoma and SH-SY5Y neuronal cells facilitates the release of GPC6 and decreases the surface level of GPC6 (Matas-Rico et al., 2016; Salgado-Polo et al., 2020). Knock-down or 
knock-out of Gpc6 induces neuronal differentiation similarly to the overexpression of GDE2, suggesting that removal of surface GPC6 by GDE2 mediates the neuronal differentiation in a cell-autonomous manner. Interestingly, a recent study showed that the plasma level of GPLD1 increases after exercise (Horowitz et al., 2020). It is notable that overexpression of GPLD1 WT but not a catalytic-dead mutant ameliorates age-related cognitive impairment and enhances adult neurogenesis in aged animals. These results underscore the significant roles of the GPI-anchored proteins and their regulation in neuronal development and function. The GPI lipase that mediates GPC4 release in astrocytes remains to be determined.

The synaptogenic function of GPC4 requires the presence of heparan sulfate side chain. These heparan sulfate side chains are attached to residues proximal to the $C$ terminus and are thought to mediate binding to GPC4 receptors (Allen et al., 2012; Farhy-Tselnicker et al., 2017; Condomitti et al., 2018). While a lipase shedding mechanism releases GPC4 intact, including the heparan sulfate modification, proteolytic shedding releases a prodomain fragment, and depending on the cleavage site, could shed GPC4 with or without heparan sulfate modification. Our results show that endogenously shed GPC4 is pulled down by a DEAE anion exchange column at similar ratios as PI-PLC lipase shed GPC4, suggesting that endogenously shed GPC4 is heparan sulfate modified and capable of known synaptogenic functions.

Given our results, we expect both lipase and protease shed GPC4 to be capable of functioning as a synaptogenic factor. However, this raises questions as to why there are multiple shedding mechanisms for GPC4 and whether the GPI anchor posttranslational modification plays a role in, or modifies GPC4 function. There could be differences in stability between lipase and protease shed GPC4 in the extracellular space. For example, unlike an ectodomain fragment shed by a protease, the core GPI structure attached to the $C$ terminus of the protein released by a GPI lipase may play a protective role against extracellular proteases, such as carboxypeptidase $\mathrm{E}$, which is secreted by astrocytes (Ji et al., 2017; Klein et al., 1992). It is also possible that the location and the upstream regulatory signals may differ between proteolytic and GPI lipase enzymes, allowing for differential control of shedding. In addition, given the heterogeneity of astrocyte subpopulations, these activities may be achieved by distinct astrocyte subtypes (John Lin et al., 2017). Future investigations will be required to determine the localization, regulation, and subtype-specific expression of the releasing enzymes.

Overall, our study demonstrates that soluble forms of GPC4 are released from the astrocyte surface and contain the heparan sulfate side chain. ADAM9 is a key enzyme that mediates the release of GPC4 from astrocytes. Since, unlike other secreted factors, GPC4 is released from the cell surface by releasing enzymes, our study will provide an opportunity in understanding the regulatory mechanism by which astrocytes promotes synapse maturation and function.

\section{References}

Allen NJ, Bennett ML, Foo LC, Wang GX, Chakraborty C, Smith SJ, Barres BA (2012) Astrocyte glypicans 4 and 6 promote formation of excitatory synapses via GluA1 AMPA receptors. Nature 486:410-414.

Bajor M, Kaczmarek L (2013) Proteolytic remodeling of the synaptic cell adhesion molecules (CAMs) by metzincins in synaptic plasticity. Neurochem Res 38:1113-1121.

Brew K, Nagase H (2010) The tissue inhibitors of metalloproteinases (TIMPs): an ancient family with structural and functional diversity. Biochim Biophys Acta 1803:55-71.

Cahoy JD, Emery B, Kaushal A, Foo LC, Zamanian JL, Christopherson KS, Xing Y, Lubischer JL, Krieg PA, Krupenko SA, Thompson WJ, Barres BA (2008) A transcriptome database for astrocytes, neurons, and oligodendrocytes: a new resource for understanding brain development and function. J Neurosci 28:264-278.

Chen R, Walter El, Parker G, Lapurga JP, Millan JL, Ikehara Y, Udenfriend S, Medof ME (1998) Mammalian glycophosphatidylinositol anchor transfer to proteins and posttransfer deacylation. Proc Natl Acad Sci USA 95:9512-9517.

Choi BR, Dobrowolski M, Sockanathan S (2021) GDE2 expression in oligodendroglia regulates the pace of oligodendrocyte maturation. Dev Dyn 250:513-526.

Christopherson KS, Ullian EM, Stokes CC, Mullowney CE, Hell JW, Agah A, Lawler J, Mosher DF, Bornstein P, Barres BA (2005) Thrombospondins are astrocyte-secreted proteins that promote CNS synaptogenesis. Cell 120:421-433.

Condomitti G, Wierda KD, Schroeder A, Rubio SE, Vennekens KM, Orlandi C, Martemyanov KA, Gounko NV, Savas JN, de Wit J (2018) An input-specific orphan receptor GPR158-HSPG interaction organizes hippocampal mossy fiber-CA3 synapses. Neuron 100:201-215.e9.

Davis S, Aldrich TH, Ip NY, Stahl N, Scherer S, Farruggella T, DiStefano PS, Curtis R, Panayotatos N, Gascan H (1993) Released form of CNTF receptor alpha component as a soluble mediator of CNTF responses. Science 259:1736-1739.

Dobrowolski M, Cave C, Levy-Myers R, Lee C, Park S, Choi BR, Xiao B, Yang W, Sockanathan S (2020) GDE3 regulates oligodendrocyte precursor proliferation via release of soluble CNTFRalpha. Development 147:dev180695.

Dowling C, Allen NJ (2018) Mice lacking glypican 4 display juvenile hyperactivity and adult social interaction deficits. Brain Plast 4:197-209.

Farhy-Tselnicker I, van Casteren ACM, Lee A, Chang VT, Aricescu AR, Allen NJ (2017) Astrocyte-secreted glypican 4 regulates release of neuronal pentraxin 1 from axons to induce functional synapse formation. Neuron 96:428-445.e13.

Feitsma K, Hausser H, Robenek H, Kresse H, Vischer P (2000) Interaction of thrombospondin-1 and heparan sulfate from endothelial cells. Structural requirements of heparan sulfate. J Biol Chem 275:9396-9402.

Fernández-Messina L, Ashiru O, Boutet P, Agüera-González S, Skepper JN, Reyburn HT, Valés-Gómez M (2010) Differential mechanisms of shedding of the glycosylphosphatidylinositol (GPI)-anchored NKG2D ligands. J Biol Chem 285:8543-8551.

Filmus J, Capurro M, Rast J (2008) Glypicans. Genome Biol 9:224.

Greco TM, Seeholzer SH, Mak A, Spruce L, Ischiropoulos H (2010) Quantitative mass spectrometry-based proteomics reveals the dynamic range of primary mouse astrocyte protein secretion. $J$ Proteome Res 9:2764-2774.

Grell M, Douni E, Wajant H, Löhden M, Clauss M, Maxeiner B, Georgopoulos S, Lesslauer W, Kollias G, Pfizenmaier K, Scheurich $P$ (1995) The transmembrane form of tumor necrosis factor is the prime activating ligand of the $80 \mathrm{kDa}$ tumor necrosis factor receptor. Cell 83:793-802.

Heise K, Oppermann H, Meixensberger J, Gebhardt R, Gaunitz F (2013) Dual luciferase assay for secreted luciferases based on Gaussia and NanoLuc. Assay Drug Dev Technol 11:244-252. 
Hettmann T, Siddiqui RA, von Langen J, Frey C, Romão MJ, Diekmann S (2003) Mutagenesis study on the role of a lysine residue highly conserved in formate dehydrogenases and periplasmic nitrate reductases. Biochem Biophys Res Commun 310:40-47.

Hong Y, Ohishi K, Inoue N, Kang JY, Shime H, Horiguchi Y, van der Goot FG, Sugimoto N, Kinoshita T (2002) Requirement of N-glycan on GPI-anchored proteins for efficient binding of aerolysin but not Clostridium septicum alpha-toxin. EMBO J 21:5047-5056.

Horowitz AM, Fan X, Bieri G, Smith LK, Sanchez-Diaz CI, Schroer AB, Gontier G, Casaletto KB, Kramer JH, Williams KE, Villeda SA (2020) Blood factors transfer beneficial effects of exercise on neurogenesis and cognition to the aged brain. Science 369:167-173.

Ji L, Wu HT, Qin XY, Lan R (2017) Dissecting carboxypeptidase E: properties, functions and pathophysiological roles in disease. Endocr Connect 6:R18-R38.

John Lin CC, Yu K, Hatcher A, Huang TW, Lee HK, Carlson J, Weston MC, Chen F, Zhang Y, Zhu W, Mohila CA, Ahmed N, Patel AJ, Arenkiel BR, Noebels JL, Creighton CJ, Deneen B (2017) Identification of diverse astrocyte populations and their malignant analogs. Nat Neurosci 20:396-405.

Kato $\mathrm{M}$, Wang $\mathrm{H}$, Kainulainen $\mathrm{V}$, Fitzgerald ML, Ledbetter S, Ornitz DM, Bernfield M (1998) Physiological degradation converts the soluble syndecan-1 ectodomain from an inhibitor to a potent activator of FGF-2. Nat Med 4:691-697.

Klein RS, Das B, Fricker LD (1992) Secretion of carboxypeptidase E from cultured astrocytes and from AtT-20 cells, a neuroendocrine cell line: implications for neuropeptide biosynthesis. J Neurochem 58:2011-2018.

Kojima T, Leone CW, Marchildon GA, Marcum JA, Rosenberg RD (1992) Isolation and characterization of heparan sulfate proteoglycans produced by cloned rat microvascular endothelial cells. J Biol Chem 267:4859-4869.

Konoshenko MY, Lekchnov EA, Vlassov AV, Laktionov PP (2018) Isolation of extracellular vesicles: general methodologies and latest trends. Biomed Res Int 2018:8545347.

Lam DK, Dang D, Flynn AN, Hardt M, Schmidt BL (2015) TMPRSS2, a novel membrane-anchored mediator in cancer pain. Pain 156:923-930.

Li Q, Cheng Z, Zhou L, Darmanis S, Neff NF, Okamoto J, Gulati G, Bennett ML, Sun LO, Clarke LE, Marschallinger J, Yu G, Quake SR, Wyss-Coray T, Barres BA (2019) Developmental heterogeneity of microglia and brain myeloid cells revealed by deep single-cell RNA sequencing. Neuron 101:207-223.e10.

Lichtenthaler SF, Lemberg MK, Fluhrer R (2018) Proteolytic ectodomain shedding of membrane proteins in mammals-hardware, concepts, and recent developments. EMBO J 37:e99456.

Linsenmeier L, Mohammadi B, Wetzel S, Puig B, Jackson WS, Hartmann A, Uchiyama K, Sakaguchi S, Endres K, Tatzelt J, Saftig P, Glatzel M, Altmeppen HC (2018) Structural and mechanistic aspects influencing the ADAM10-mediated shedding of the prion protein. Mol Neurodegener 13:18.

Matas-Rico E, van Veen M, Leyton-Puig D, van den Berg J, Koster J, Kedziora KM, Molenaar B, Weerts MJA, de Rink I, Medema $\mathrm{RH}$, Giepmans BNG, Perrakis A, Jalink K, Versteeg R, Moolenaar WH (2016) Glycerophosphodiesterase GDE2 promotes neuroblastoma differentiation through glypican release and is a marker of clinical outcome. Cancer Cell 30:548-562.

Melo SA, Luecke LB, Kahlert C, Fernandez AF, Gammon ST, Kaye J, LeBleu VS, Mittendorf EA, Weitz J, Rahbari N, Reissfelder C, Pilarsky C, Fraga MF, Piwnica-Worms D, Kalluri R (2015) Glypican1 identifies cancer exosomes and detects early pancreatic cancer. Nature 523:177-182.
Mollinedo F, Gajate C, Martín-Santamaría S, Gago F (2004) ET-18$\mathrm{OCH} 3$ (edelfosine): a selective antitumour lipid targeting apoptosis through intracellular activation of Fas/CD95 death receptor. Curr Med Chem 11:3163-3184.

Moss ML, Powell G, Miller MA, Edwards L, Qi B, Sang QXA, De Strooper B, Tesseur I, Lichtenthaler SF, Taverna M, Zhong JL, Dingwall C, Ferdous T, Schlomann U, Zhou P, Griffith LG, Lauffenburger DA, Petrovich R, Bartsch JW (2011) ADAM9 inhibition increases membrane activity of ADAM10 and controls $\alpha$-secretase processing of amyloid precursor protein. $\mathrm{J}$ Biol Chem 286:40443-40451.

Muller GA (2018) The release of glycosylphosphatidylinositol-anchored proteins from the cell surface. Arch Biochem Biophys 656:118.

Nagappan-Chettiar S, Johnson-Venkatesh EM, Umemori H (2017) Activity-dependent proteolytic cleavage of cell adhesion molecules regulates excitatory synaptic development and function. Neurosci Res 116:60-69.

Park S, Lee C, Sabharwal P, Zhang M, Meyers CL, Sockanathan S (2013) GDE2 promotes neurogenesis by glycosylphosphatidylinositol-anchor cleavage of RECK. Science 339:324-328.

Reiss K, Maretzky T, Ludwig A, Tousseyn T, de Strooper B, Hartmann D, Saftig P (2005) ADAM10 cleavage of N-cadherin and regulation of cell-cell adhesion and beta-catenin nuclear signalling. EMBO J 24:742-752.

Salgado-Polo F, van Veen M, van den Broek B, Jalink K, Leyton-Puig D, Perrakis A, Moolenaar WH, Matas-Rico E (2020) Sequence-dependent trafficking and activity of GDE2, a GPI-specific phospholipase promoting neuronal differentiation. J Cell Sci 133:jcs235044.

Sanz RL, Ferraro GB, Kacervosky J, Salesse C, Gowing E, Hua L, Rambaldi I, Beaubien F, Holmbeck K, Cloutier JF, Lévesque M, Murai K, Fournier AE (2018) MT3-MMP promotes excitatory synapse formation by promoting Nogo-66 receptor ectodomain shedding. J Neurosci 38:518-529.

Shin DJ, Cho D, Kim YR, Rhee JH, Choy HE, Lee JJ, Hong Y (2006) Diagnosis of paroxysmal nocturnal hemoglobinuria by fluorescent clostridium septicum alpha toxin. J Mol Microbiol Biotechnol 11:20-27.

van Veen M, Matas-Rico E, van de Wetering $K$, Leyton-Puig $D$, Kedziora KM, De Lorenzi V, Stijf-Bultsma Y, van den Broek B, Jalink K, Sidenius N, Perrakis A, Moolenaar WH (2017) Negative regulation of urokinase receptor activity by a GPI-specific phospholipase C in breast cancer cells. Elife 6:e23649.

Vidal M (2020) Exosomes and GPI-anchored proteins: judicious pairs for investigating biomarkers from body fluids. Adv Drug Deliv Rev 161-162:110-123.

Webber CA, Hocking JC, Yong VW, Stange CL, McFarlane S (2002) Metalloproteases and guidance of retinal axons in the developing visual system. J Neurosci 22:8091-8100.

Wilson ES, Newell-Litwa K (2018) Stem cell models of human synapse development and degeneration. Mol Biol Cell 29:2913-2921.

Zhang Y, Chen K, Sloan SA, Bennett ML, Scholze AR, O'Keeffe S, Phatnani HP, Guarnieri P, Caneda C, Ruderisch N, Deng S, Liddelow SA, Zhang C, Daneman R, Maniatis T, Barres BA, Wu JQ (2014) An RNA-sequencing transcriptome and splicing database of glia, neurons, and vascular cells of the cerebral cortex. J Neurosci 34:11929-11947.

Zhou CY, Dong YP, Sun X, Sui X, Zhu H, Zhao YQ, Zhang YY, Mason C, Zhu Q, Han SX (2018) High levels of serum glypican-1 indicate poor prognosis in pancreatic ductal adenocarcinoma. Cancer Med 7:5525-5533. 